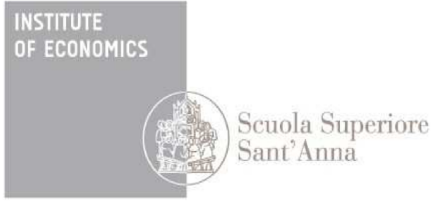

LEM | Laboratory of Economics and Management

Institute of Economics

Scuola Superiore Sant'Anna

Piazza Martiri della Libertà, 33 - 56127 Pisa, Italy ph. +3905088.33 .43$

institute.economics@sssup.it

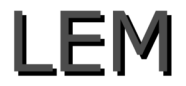

Working Paper Series

\title{
Fat-Tail Distributions and Business-Cycle Models
}

\author{
Guido Ascari ^ \\ Giorgio Fagiolo ${ }^{\circ}$ \\ Andrea Roventini *
}

University of Pavia, Italy

- Scuola Superiore Sant'Anna, Pisa, Italy

* University of Paris Ouest Nanterre La Defense, France; University of

Verona, Italy ; Scuola Superiore Sant'Anna, Pisa, Italy; and OFCE-Science Po, Nice, France 


\title{
Fat-Tail Distributions and Business-Cycle Models*
}

\author{
Guido Ascari $^{\dagger} \quad$ Giorgio Fagiolo $^{\ddagger} \quad$ Andrea Roventini ${ }^{\S}$ \\ January 17, 2012
}

\begin{abstract}
Recent empirical findings suggest that macroeconomic variables are seldom normally distributed. For example, the distributions of aggregate output growth-rate time series of many OECD countries are well approximated by symmetric exponential-power (EP) densities, with Laplace fat tails. In this work, we assess whether Real Business Cycle (RBC) and standard medium-scale New-Keynesian (NK) models are able to replicate this statistical regularity. We simulate both models drawing Gaussian- vs Laplace-distributed shocks and we explore the statistical properties of simulated time series. Our results cast doubts on whether RBC and NK models are able to provide a satisfactory representation of the transmission mechanisms linking exogenous shocks to macroeconomic dynamics.
\end{abstract}

Keywords: Growth-Rate Distributions, Normality, Fat Tails, Time Series, ExponentialPower Distributions, Laplace Distributions, DSGE Models, RBC Models.

JEL Classification: C1, E3

${ }^{*}$ Thanks to Alice Albonico, Jean-Luc Gaffard, Mauro Napoletano, Francesco Saraceno, Alessandro Spelta, Harald Uhlig, Philippe Weil, and participants to the Computing in Economics and Finance Conference 2011 (San Francisco) and the OFCE Seminar Series, Sciences Po (Paris and Nice), for their stimulating and helpful comments. All usual disclaimers apply.

${ }^{\dagger}$ University of Pavia, Italy. Email: guido.ascari@unipv.it

${ }^{\ddagger}$ Sant’Anna School of Advanced Studies, Pisa, Italy. Email: giorgio.fagiolo@sssup.it

$\S$ University Paris Ouest Nanterre La Defense, France, University of Verona, Italy, Sant'Anna School of Advanced Studies, Pisa, Italy, OFCE, Sciences Po, Nice France. Email: andrea.roventini@univr.it 


\section{Introduction}

Gaussian assumptions are extensively employed in theoretical and applied macroeconomics. An increasing number of recent studies, however, have started to question this practice. For example, Christiano (2007) criticizes using normal likelihood functions. He shows that the distributions of the residuals of standard VAR analyses display marked excess kurtosis, concluding that "the evidence against the normality assumption is substantial" (Christiano, 2007, p. 145).

Similarly, Mishkin (2011) warns against the use of Gaussian shocks in quantitative studies of optimal monetary policy, arguing that in reality "the distribution of shocks hitting the economy is more complex" and "may exhibit excess kurtosis, that is, tail risk" (p. 24). Empirical findings strongly support Mishkin's argument. For instance, growth rates of macroeconomic variables are seldom normally distributed, no matter if they are observed over a cross section or along a time series. More specifically, Fagiolo et al. (2008) show that, in the majority of OECD countries, the distribution of within-country time-series output growth rates - properly depurated from autocorrelation, outliers, and additional structure - is well approximated by symmetric exponential-power (EP) densities with Laplace tails, persistently fatter than Gaussian ones. In other words, extreme output changes typically occur more frequently than implied by a normal distribution. ${ }^{1}$

Non normality and fat tails do not characterize only output growth rates. In Table 1, we summarize the statistical properties of growth-rate distributions for some important macroeconomic U.S. time series such as real GDP (Y), consumption (C), investment (I), employment (E), inflation $(\mathrm{P})$, and real wage $(\mathrm{W})$. We report their second, third and fourth moments, together with a battery of normality tests for the null hypothesis that data are normally distributed. Both the large kurtosis figures and normality-test p-values suggest that growth-rate distributions of U.S. time series are not normal and exhibit fat tails. Note also that skewness is quite mild in all series.

In this paper, we ask if existing standard macroeconomic models are able to replicate this statistical regularity. In particular, we focus on two standard workhorse models: the Real Business Cycle (RBC) model and the standard medium-scale New Keynesian (NK) model. ${ }^{2}$ There exist two basic sources allowing for fat-tail emergence in RBC or NK models. First, a given model can endogenously generate fat tails because of its structural assumptions, even if it is hit by purely Gaussian uncorrelated shocks. In this case, fat-tail distributed timeseries arise via the endogenous transmission mechanism embodied in the model. Second, fat tails could just be the result of exogenous stochastic disturbances, whose distribution is nonGaussian. Obviously, such an approach would require to find an appropriate distributions for the exogenous shocks. Following Fagiolo et al. (2008), we suggest below that EP densities

\footnotetext{
${ }^{1}$ Interestingly, fat-tail output growth-rate distributions emerge also for cross-section data, both at the level of countries (Canning et al., 1998; Lee et al., 1998; Castaldi and Dosi, 2009), industries (Castaldi and Sapio, 2008) and firms (Bottazzi and Secchi, 2003; Fu et al., 2005).

${ }^{2}$ The former is a basic RBC model: the text-book neoclassical growth model with technology shocks. The latter is a version of the standard medium-scale model used in various papers (cf., for example, Smets and Wouters, 2003; Christiano et al., 2005; Schmitt-Grohé and Uribe, 2006). Shocks are modeled as in Smets and Wouters (2003).
} 
with Laplace tails are a very good candidate. This is justified by the observation that Laplace distributions have been shown to well-proxy the properties of U.S. output growth-rate time series distributions. Furthermore, as we discuss in what follows, growth-rate distributions of other U.S. macroeconomic variables are close to be Laplacian.

This paper explores both sources of fat-tail emergence in macroeconomics by simulating RBC and NK models, using shocks drawn from either a Gaussian or an EP/Laplace distribution. Note that, since the model has to be non-linear to endogenously produce non-Gaussian distributions from Gaussian shocks, we shall simulate both RBC and NK models using a secondorder approximation.

We try to answer a simple research question: can the two workhorse macroeconomic models replicate the statistical regularity regarding higher moments of growth-rate macroeconomic time-series distributions? In particular, can such models generate fat tails? Our results suggest a negative answer. We show that, in the RBC case, simulated time-series distributions simply mirror the ones of the shocks hitting the model. This is because the RBC model lacks an internal shock propagation mechanism (see Cogley and Nason, 1995). In other words, it does not add structure to the nature of the shocks, thus acting like a neutral filter. This implies that the RBC model is able to replicate fat tails exogenously, but not endogenously. The NK model, instead, never generates fat-tail growth-rate distributions, either endogenously or exogenously. What is more, the NK structure points in the wrong direction: even if the model is hit by fat-tail shocks, it delivers quasi-normal growth-rate distributions for simulated macroeconomic time-series.

The idea that the economy can be hit by positive-probability big shocks is not new in macroeconomics (see, e.g., Rietz, 1988; Barro, 2006). The literature on fat tails and macroeconomic modeling, however, is still in its infancy, even if it may gain more attention as a consequence of the global financial crisis. Of course, there may be alternative sources of non-normality in the data that we do not investigate in this paper. Non-normality in DSGE models may arise from stochastic volatility as in Fernández-Villaverde and Rubio-Ramírez (2007) and Justiniano and Primiceri (2008), who introduce low-frequency movements in volatility to explain the source of the Great Moderation period. Moreover, Posch (2009) shows that non-normality can also result from the presence of jumps in aggregate productivity in a standard neoclassical growth model.

The rest of the paper is organized as follows. Section 2 discusses the methodology that we employ in our exercises. Results are presented in Section 3. Finally, Section 4 concludes.

\section{Methodology}

We begin by comparing growth-rate distributions of quarterly U.S. time series of real GDP $(\mathrm{Y})$, consumption $(\mathrm{C})$, investment $(\mathrm{I})$, employment $(\mathrm{E})$, inflation $(\mathrm{P})$, and real wage $(\mathrm{W})$, with simulated distributions for the same variables as generated by RBC and NK models under different assumptions about the underlying shock distributions. Observed U.S. series range 
from 1948Q1 to 2010Q4 (251 observations) and are drawn from the St. Louis Federal Reserve Economic Data (FRED) database ${ }^{3}$. We take second-order approximations of RBC and NK models and we perform a Monte Carlo analysis, generating 1000 series of 1000 observations for each macroeconomic variable.

We play with two scenarios as far as shocks are concerned. In the first scenario, we draw i.i.d. shocks from a standard Gaussian distribution. In the second one, shocks are i.i.d. and Laplace distributed, with zero mean and unit standard deviation. Their density thus reads:

$$
p(z)=\frac{\exp \{-\sqrt{2}|z|\}}{\sqrt{2}} .
$$

As mentioned above, Laplace distributions are good first-pass proxies for empirically-observed growth-rate time series in the U.S. and OECD countries. Therefore we shall begin by this simplifying assumption, leaving for a future investigation a more precise variable-specific shock generating mechanism.

In our analysis, we compute growth rates $g(t)$ for any variable as:

$$
g(t)=\frac{X(t)-X(t-1)}{X(t-1)} \cong x(t)-x(t-1)=(1-L) x(t)
$$

where $X(t)$ is any series employed in this study at time/quarter $t, x(t)=\ln [X(t)]$ and $L$ is the lag operator. Given the time interval $T_{n}=\left\{t_{1}, \ldots, t_{n}\right\}$ over which we observe our data, we define the time-series distribution of growth rates as:

$$
G_{T_{n}}=\left\{g(t), t \in T_{n}\right\}
$$

To compare simulated and observed time-series distributions, we employ a parametric approach. More precisely, following Fagiolo et al. (2008, 2009), we fit growth-rate distributions with exponential-power (EP) densities (for details see, Agrò, 1995; Bottazzi and Secchi, 2003). The EP probability density function reads:

$$
f(x ; b, a, m)=\frac{1}{2 a b^{\frac{1}{b}} \Gamma\left(1+\frac{1}{b}\right)} e^{-\frac{1}{b}\left|\frac{x-m}{a}\right|^{b}},
$$

where $a>0, b>0$ and $\Gamma(\cdot)$ is the Gamma function.

The EP is a generalization of a Gaussian random variable, and it is fully characterized by three parameters: a location parameter $m$, a scale parameter $a$ and a shape parameter $b$. The location parameter controls for the mean of the distribution, whereas the scale parameter is proportional to the absolute deviation ${ }^{4}$. The parameter $b$ determines the fatness of the tails: the larger $b$, the thinner the tails. In particular, as visualized in Figure 1 , if $b=2$, the EP distribution reduces to a Gaussian, whereas if $b=1$, one recovers a Laplace (with unit standard deviation if $a=1 / \sqrt{2}$ ).

\footnotetext{
${ }^{3}$ Freely available online at http://research.stlouisfed.org/fred2/.

${ }^{4}$ On the links between moments and parameters of the EP distribution, see Bottazzi (2004).
} 
The EP distribution allows one to precisely measure how far the empirical distribution is from the normal and Laplace benchmarks. Note also that the EP density is characterized by exponentially-shaped tails, and thus it has finite moments of any order. This is important, as in many financial applications one typically deals with heavy-tails distributions whose higher moments (and sometimes even the mean) do not converge (Embrechts et al., 1997). Macroeconomic growth-rate time-series distributions, conversely, almost always possess finite moments. Therefore, we do not expect, at least in principle, RBC and NK macroeconomic model to badly behave with such smooth shock distributions.

We fit empirical and simulated distributions with the EP density in Eq. (4) by jointly estimating the three parameters via maximum likelihood $(\mathrm{ML})^{5}$. Next, we compare EP-density parameter estimates obtained from the data with the mean of the ones obtained by fitting the EP to each Montecarlo simulated time-series. To do so, we calibrate the RBC and NK as in the standard literature (see, e.g., Smets and Wouters, 2003), properly adjusting the standard deviations of simulated shocks in either scenarios. Furthermore, before carrying out ML estimation we subtract the mean from all time series. Since we are mainly interested in the tail parameter $b$, this does not affect our estimates, but improves overall estimation efficiency. Note also that Montecarlo parameter-estimate distributions are unimodal and quite symmetric. Therefore, the mean is a good proxy for the aggregate behavior of our models. No dramatic difference is detected if instead of taking the mean of estimates, one simply pools all zero-mean time-series together and estimates a unique $b$ parameter by fitting the EP to the pooled sample.

\section{Results}

Table 1 suggests that empirical growth-rate distributions of U.S. series are characterized by fat tails. This is confirmed by our EP fits. In line with previous empirical evidence (Fagiolo et al., 2008, 2009), the GDP growth-rate distribution is well approximated by a Laplace density (cf. Figure 2 and Table 2). Moreover, growth-rate distributions of all other U.S. time series are markedly non-Gaussian: estimates of the shape parameter $(\hat{b})$ range from a maximum of 1.51 for real wage to a minimum of 0.95 for inflation. These results indicate that the presence of quasi-Laplacian fat tails in growth-rate distributions is a pervasive statistical regularity for U.S. macroeconomic time series. We now turn to assess whether the RBC and the NK models are able to account for this empirical evidence.

The RBC model. Our main results for the RBC model are summarized in Tables 3 and 4 . To begin with, note that the second and the third moment of RBC simulated time-series distributions do not substantially change if shocks are drawn from a Gaussian or Laplace distribution. ${ }^{6}$ This is not the case for the fourth moment: in presence of Laplace shocks, growth-

\footnotetext{
${ }^{5}$ We employ the package SUBBOTOOLS, freely available online at http://cafim.sssup.it/software.html. See Bottazzi (2004) for details. See also Bottazzi and Secchi (2006) for other theoretical and computational issues concerning this procedure.

${ }^{6}$ Relative standard deviations, autocorrelations and crosscorrelations with output are not affected by the source of the shocks either.
} 
rate series strongly exhibit excess kurtosis, whereas Gaussian shocks imply fourth moments that lie very close to 3 , i.e. the normal benchmark. This result is confirmed by normality tests. In presence of Gaussian shocks, the null hypothesis of normality is not rejected at $5 \%$ confidence level in at least $80 \%$ of the series generated by the model. On the contrary, with Laplace shocks, the null hypothesis of normality is always rejected.

Fitting EP densities to the series reveals that the assumptions concerning the nature of shocks have a very strong impact on the growth-rate distributions of the series generated by the RBC model (see Table 4). If shocks are Gaussian, estimates of the shape parameter $\hat{b}$ oscillate around 2 for all the simulated time series, hinting to Gaussian tails. At the opposite, if shocks are drawn from a Laplace, the $\hat{b}$ 's are slightly larger than 1 , the hallmark of Laplacian tails. This behavior is well captured by the top panels of Figure 3, where, for Montecarlopooled output growth-rate time series generated by the RBC model under alternative shock assumptions, we plot binned densities against the maximum-likelihood fitted ones (in a semi-log scale). These results, well in line with results in Cogley and Nason (1995), suggest that the propagation mechanism is almost neutral in RBC models: distributional properties of growthrate time series completely reflect those of the underlying shocks (see also Rotemberg and Woodford, 1996).

The NK model. The first interesting moments of simulated time series generated by the NK model are reported in Table 5. As for the RBC model, second and third moments - as well as relative standard deviation, autocorrelations and cross-correlations with output, not reported here - are not dramatically affected by shock distributions. Conversely, kurtosis changes when we move from Gaussian to Laplace shocks. However, changes in kurtosis are less pronounced in the NK than in the RBC model. In presence of Gaussian shocks, time-series distributions still exhibit quasi-Gaussian kurtosis, whereas if shocks are Laplace distributed, kurtosis ranges from 3.2 (investment) to 5 (employment). As it happens in the RBC model, normality-test outcomes are also influenced by the distribution of the shocks. However, the percentage of cases for which normality is rejected is lower than in the RBC case. Furthermore, for investment and real wages the null hypothesis of normality cannot be rejected at $5 \%$ confidence level for most of Montecarlo replications.

All that is reflected in the estimation of the $\hat{b}$ 's, see Table 6 . In presence of Gaussian shocks, growth-rate time-series are normally-distributed, as $\hat{b}$ 's lie very close to 2 . This is in line with $\mathrm{RBC}$ results above. If shocks become Laplace-distributed, simulated growth-rate distributions display fatter tails. However, in contrast with the RBC model, tails are still far from the Laplace benchmark. The minimum estimated $\hat{b}$ is 1.27 for employment, while $\hat{b}$ is very close to 2 for investment and real wage.

The NK model is therefore unable to reproduce the statistical regularity concerning fat-tail growth-rate time-series distributions, even in presence of Laplace-distributed (fat-tail) shocks, cf. also the bottom panel of Figure 3. Rather than inducing stronger non-linearities, it seems that the ensemble of real and nominal rigidities, added in the NK model upon the bare-bone structure of the RBC model tends to smooth time series of exogenous, fat-tail Laplacian shocks. 
Robustness analysis. The foregoing results are robust to a number of additional tweaks. For example, we have modified the approximation method employed in the model (i.e., employing first-order, linear approximations instead of second-order ones), changed the econometric length of Montecarlo samples (i.e., generating time series with a length equal to the empirical ones 251 observations), and computed growth rates as $g(t)=\Delta X(t) / X(t-1)-1$, to check if the implicit approximation in log-difference approximations could have affected our results. In all the exercises we have performed, results were not substantially altered, hinting to a substantial robustness of our main insights. ${ }^{7}$

\section{Concluding Remarks}

Observed growth-rate distributions of U.S. macroeconomic time-series (output, consumption, investment, inflation, employment and real wage) strongly depart from the Gaussian benchmark and are well proxied by EP densities with Laplacian tails.

This paper asks whether standard workhorse macroeconomic models of business cycle, such as the basic RBC and NK models, are able to replicate this statistical regularity. The answer is far from encouraging.

We find that neither the RBC nor the NK model can generate fat-tail time-series distributions if the are hit by i.i.d. Gaussian shocks. This implies that endogenous transmission mechanisms embedded in both RBC and NK models are not able to reproduce fat tails from normal disturbances. Conversely, when the RBC model is subject to i.i.d. Laplace-distributed shocks, it tends to generate Laplace-distributed growth-rate distributions for simulated time series. This results from quasi-linearity of the RBC model and from neutrality to shocks of its endogenous transmission mechanisms. In other words, the growth-rate distribution of the main series generated by the model have the same shape of the shock distribution that is fed into the model. On the contrary, the NK structure points in the wrong direction. When shocks are Laplacian, the NK model tends to deliver growth-rate distributions whose tails that are slightly fatter than Gaussian ones. This suggests that the endogenous transmission mechanism of the NK model induced by the several real and nominal rigidities hardwired into the model is actually smoothing extremal events generated by the fat tails of Laplace disturbances.

Ideally, one would like a model that can endogenously reproduce fat tails from Gaussian shocks, thanks to transmission mechanisms built into the model. This might allow for a better understanding of how alternative mechanisms lead to the endogenous emergence of highprobability large events. Our results seem to suggest that the two workhorse models employed in current macroeconomic business-cycle analysis are not able to fulfill this expectations. Therefore, further research in this field is required to bridge more firmly business-cycle models to empirical statistical regularities concerning growth-rate time-series distributions of macroeconomic variables.

\footnotetext{
${ }^{7}$ Data, codes and results are fully available from the authors upon request.
} 


\section{References}

Agrò, G. (1995), "Maximum Likelihood Estimation for the Exponential Power Function Parameters", Communications in Statistics, 24: 523-536.

Barro, R. J. (2006), "Rare Disasters and Asset Markets in the Twentieth Century", Quarterly Journal of Economics, 121: 823-866.

Bottazzi, G. (2004), "SUBBOTOOLS User's Manual", Working Paper 2004/14, Laboratory of Economics and Management (LEM), Sant'Anna School of Advanced Studies, Pisa, Italy.

Bottazzi, G. and A. Secchi (2003), "Common Properties and Sectoral Specificities in the Dynamics of U.S. Manufacturing Firms", Review of Industrial Organization, 23: 217-32.

Bottazzi, G. and A. Secchi (2006), "Maximum Likelihood Estimation of the Symmetric and Asymmetric Exponential Power Distribution", Working Paper 2006/19, Laboratory of Economics and Management (LEM), Sant'Anna School of Advanced Studies, Pisa, Italy.

Canning, D., L. A. N. Amaral, Y. Lee, M. Meyer and H. E. Stanley (1998), "Scaling the Volatility of GDP Growth Rates", Economic Letters, 60: 335-41.

Castaldi, C. and G. Dosi (2009), "The patterns of output growth of firms and countries: Scale invariances and scale specificities", Empirical Economics, 37: 475-495.

Castaldi, C. and S. Sapio (2008), "Growing like mushrooms? Sectoral evidence from four large European economies", Journal of Evolutionary Economics, 18: 509-527.

Christiano, L. G., M. Eichenbaum and C. L. Evans (2005), "Nominal Rigidities and the Dynamic Effects of a Shock to Monetary Policy", Journal of Political Economy, 113: 1-45.

Christiano, L. J. (2007), "Comment on 'On the Fit of New Keynesian Models' by Del Negro, Schorfheide, Smets and Wouters", Journal of Business and Economic Statistics, 25: 143-151.

Cogley, T. and J. M. Nason (1995), "Output Dynamics in Real-Business-Cycle Models", American Economic Review, 85: 492-511.

Embrechts, P., C. Klüppelberg and T. Mikosch (1997), Modelling extremal events for insurance and finance, Springer.

Fagiolo, G., M. Napoletano, M. Piazza and A. Roventini (2009), "Detrending and the Distributional Properties of U.S. Output Time Series", Economics Bulletin, 29: 3155-3161.

Fagiolo, G., M. Napoletano and A. Roventini (2008), "Are Output Growth-Rate Distributions Fat-Tailed? Some Evidence from OECD Countries", Journal of Applied Econometrics, 23: 639-669.

Fernández-Villaverde, J. and J. F. Rubio-Ramírez (2007), "Estimating Macroeconomic Models: A Likelihood Approach", Review of Economic Studies, 74: 1059-1087.

Fu, D., F. Pammolli, S. Buldyrev, M. Riccaboni, K. Matia, K. Yamasaki and H. Stanley (2005), "The growth of business firms: Theoretical framework and empirical evidence", Proceedings of the Nationaly Academy of Science, 102.

Justiniano, A. and G. Primiceri (2008), "The Time-Varying Volatility of Macroeconomic Fluctuations", American Economic Review, 98: 604-641. 
Lee, Y., L. A. N. Amaral, D. Canning, M. Meyer and H. E. Stanley (1998), "Universal Features in the Growth Dynamics of Complex Organizations", Physical Review Letters, 81: 3275-8.

Mishkin, F. S. (2011), "Monetary Policy Strategy: Lessons from the Crisis", Working Paper 16755, NBER.

Posch, O. (2009), "Structural Estimation of Jump-Diffusion Processes in Macroeconomics", mimeo, Aarhus University.

Rietz, T. A. (1988), "The Equity Risk Premium: A Solution", Journal of Monetary Economics, 22: $1002-1037$.

Rotemberg, J. and M. Woodford (1996), "Real-Business-Cycle Models and Forecastable Movements in Output, Hours, and Consumption", American Economic Review, 86: 71-89.

Schmitt-Grohé, S. and M. Uribe (2006), "Optimal Fiscal and Monetary Policy in a MediumScale Macroeconomic Model", in M. Gertler and K. Rogoff, (eds.), NBER Macroeconomics Annual, Cambridge, MA: MIT Press, 383-425.

Smets, F. and R. Wouters (2003), "An Estimated Dynamic Stochastic General Equilibrium Model of the Euro Area", Journal of the European Economic Association, 1: 1123-1175. 


\begin{tabular}{ccccccc} 
Series & Std. Dev. & Skewness & Kurtosis & $\begin{array}{c}\text { JB } \\
\text { test }\end{array}$ & $\begin{array}{c}\text { Lilliefors } \\
\text { test }\end{array}$ & $\begin{array}{c}\text { AD } \\
\text { test }\end{array}$ \\
\hline Y & 0.0099 & -0.0834 & 4.2402 & $16.3764^{* * *}$ & $0.0707^{* * *}$ & $1.9232^{* * *}$ \\
C & 0.0084 & -0.4250 & 8.3367 & $305.4176^{* * *}$ & $0.0954^{* * *}$ & $3.0373^{* * *}$ \\
I & 0.0530 & -0.3893 & 4.8973 & $43.9859^{* * *}$ & $0.0807^{* * *}$ & $1.8682^{* * *}$ \\
E & 0.0067 & -0.4032 & 3.9027 & $15.3222^{* * *}$ & $0.0728^{* * *}$ & $1.8521^{* * *}$ \\
W & 0.0083 & 0.7511 & 4.0603 & $35.3592^{* * *}$ & $0.0641^{* *}$ & $1.8128^{* * *}$ \\
P & 0.0079 & -0.5724 & 10.0876 & $539.0752^{* * *}$ & $0.1020^{* * *}$ & $4.7237^{* * *}$ \\
\hline
\end{tabular}

Table 1: U.S. growth-rate time series: summary statistics. Legend: (Y) real GDP, (C) consumption, (I) investment, (E) employment, (P) inflation, (W) real wage; (JB): Jarque-Bera test; (AD): Anderson-Darling test; $(* *)$ : Significant at $5 \%$ level. $(*)$ : Significant at $1 \%$ level.

\begin{tabular}{ccccc} 
& \multicolumn{2}{c}{$\widehat{b}$} & \multicolumn{2}{c}{$\widehat{a}$} \\
Series & Par. & Std. Err. & Par. & Std. Err. \\
\hline Y & 1.050 & 0.007 & 0.008 & 0.124 \\
C & 1.200 & 0.042 & 0.010 & 0.146 \\
I & 1.090 & 0.006 & 0.008 & 0.131 \\
E & 1.360 & 0.006 & 0.004 & 0.171 \\
W & 1.510 & 0.007 & 0.004 & 0.197 \\
P & 0.954 & 0.005 & -0.000 & 0.111 \\
\hline
\end{tabular}

Table 2: U.S. growth-rate distributions: Estimated EP parameters. Legend: (Y) real GDP, (C) consumption, (I) investment, (E) employment, (P) inflation, (W) real wage. 


\begin{tabular}{|c|c|c|c|c|c|c|}
\hline Series & Std. Dev. & Skewness & Kurtosis & $\begin{array}{l}\text { JB } \\
\text { test }\end{array}$ & $\begin{array}{c}\text { Lilliefors } \\
\text { test }\end{array}$ & $\begin{array}{l}\text { AD } \\
\text { test }\end{array}$ \\
\hline \multicolumn{7}{|c|}{ Gaussian shocks } \\
\hline $\mathrm{Y}$ & 0.0176 & -0.0071 & 2.9963 & 0.0460 & 0.0610 & 0.0570 \\
\hline $\mathrm{C}$ & 0.0044 & 0.0278 & 2.9948 & 0.0570 & 0.0510 & 0.0580 \\
\hline I & 0.0579 & -0.0250 & 3.0972 & 0.1370 & 0.0580 & 0.0690 \\
\hline $\mathrm{E}$ & 0.0136 & -0.0057 & 2.9973 & 0.0500 & 0.0510 & 0.0480 \\
\hline \multicolumn{7}{|c|}{ Laplace shocks } \\
\hline $\mathrm{Y}$ & 0.0176 & -0.0221 & 5.7803 & 1 & 1 & 0.9940 \\
\hline $\mathrm{C}$ & 0.0044 & 0.04659 & 5.4543 & 1 & 1 & 0.9950 \\
\hline $\mathrm{I}$ & 0.0579 & -0.2023 & 6.0382 & 1 & 1 & 0.9880 \\
\hline $\mathrm{E}$ & 0.0136 & -0.0314 & 5.5816 & 1 & 1 & 0.9960 \\
\hline
\end{tabular}

Table 3: Simulated growth-rate distributions in the Real Business Cycle model (second-order approximation): summary statistics. (JB): Jarque-Bera test; (AD): Anderson-Darling test. Normality tests report the percentage of series for which the normality hyphotesis cannot be accepted at a 5\% confidence level. Legend: (Y) real GDP, (C) consumption, (I) investment, (E) employment.

$\widehat{b} \quad \widehat{a}$

Series Par. Std. Err. Par. Std. Err.

\begin{tabular}{|c|c|c|c|c|}
\hline \multicolumn{5}{|c|}{ Gaussian shocks } \\
\hline $\mathrm{Y}$ & 2.0121 & 0.1416 & 0.0177 & 0.0006 \\
\hline $\mathrm{C}$ & 2.018 & 0.1422 & 0.0044 & 0.0001 \\
\hline I & 1.934 & 0.1345 & 0.0571 & 0.0020 \\
\hline $\mathrm{E}$ & 2.0097 & 0.1414 & 0.0136 & 0.0005 \\
\hline \multicolumn{5}{|c|}{ Laplace shocks } \\
\hline $\mathrm{Y}$ & 1.0587 & 0.0627 & 0.0129 & 0.0005 \\
\hline $\mathrm{C}$ & 1.1579 & 0.0700 & 0.0034 & 0.0001 \\
\hline I & 1.0777 & 0.0641 & 0.0427 & 0.0017 \\
\hline $\mathrm{E}$ & 1.1181 & 0.0671 & 0.0103 & 0.0004 \\
\hline
\end{tabular}

Table 4: Simulated growth-rate distributions in the Real Business Cycle model (second-order approximation): Estimated EP parameters. Legend: (Y) real GDP, (C) consumption, (I) investment, (E) employment. 


\begin{tabular}{|c|c|c|c|c|c|c|}
\hline Series & Std. Dev. & Skewness & Kurtosis & $\begin{array}{l}\text { JB } \\
\text { test }\end{array}$ & $\begin{array}{c}\text { Lilliefors } \\
\text { test }\end{array}$ & $\begin{array}{l}\text { AD } \\
\text { test }\end{array}$ \\
\hline \multicolumn{7}{|c|}{ Gaussian shocks } \\
\hline $\mathrm{Y}$ & 0.0045 & -0.0282 & 2.9980 & 0.0760 & 0.0640 & 0.0690 \\
\hline $\mathrm{C}$ & 0.0018 & 0.0041 & 2.9963 & 0.0780 & 0.0670 & 0.0750 \\
\hline $\mathrm{I}$ & 0.0045 & -0.0408 & 2.9769 & 0.1840 & 0.1150 & 0.1680 \\
\hline $\mathrm{E}$ & 0.0093 & -0.0020 & 2.9971 & 0.0540 & 0.0520 & 0.0490 \\
\hline W & 0.0019 & -0.0256 & 2.9943 & 0.0920 & 0.0600 & 0.0740 \\
\hline $\mathrm{P}$ & 0.0004 & -0.0232 & 3.0091 & 0.0690 & 0.0530 & 0.0640 \\
\hline \multicolumn{7}{|c|}{ Laplace shocks } \\
\hline $\mathrm{Y}$ & 0.0044 & -0.0132 & 3.7428 & 0.8880 & 0.3950 & 0.6550 \\
\hline $\mathrm{C}$ & 0.0018 & 0.0163 & 4.3879 & 0.9990 & 0.9250 & 0.9910 \\
\hline $\mathrm{I}$ & 0.0045 & -0.0472 & 3.1965 & 0.3820 & 0.1930 & 0.2710 \\
\hline $\mathrm{E}$ & 0.0093 & 0.0078 & 4.9904 & 1 & 0.9990 & 0.9960 \\
\hline $\mathrm{W}$ & 0.0019 & -0.0277 & 3.2570 & 0.3770 & 0.1270 & 0.2000 \\
\hline $\mathrm{P}$ & 0.0004 & -0.0517 & 4.0528 & 0.9830 & 0.7360 & 0.9190 \\
\hline
\end{tabular}

Table 5: Simulated growth-rate distributions in the NK model (second-order approximation): summary statistics. (JB): Jarque-Bera test; (AD): Anderson-Darling test. Normality tests report the percentage of series for which the normality hypothesis cannot be accepted at a 5\% confidence level. Legend: (Y) real GDP, (C) consumption, (I) investment, (E) employment, (P) inflation, (W) real wage.

$\widehat{b} \quad \widehat{a}$

Series Par. Std. Err. Par. Std. Err.

\begin{tabular}{lcccc}
\hline \multicolumn{5}{l}{ Gaussian shocks } \\
Y & 2.0141 & 0.1418 & 0.0045 & 0.0002 \\
$\mathrm{C}$ & 2.0142 & 0.1418 & 0.0018 & 0.0001 \\
$\mathrm{I}$ & 2.0418 & 0.1445 & 0.0045 & 0.0002 \\
$\mathrm{E}$ & 2.0131 & 0.1417 & 0.0093 & 0.0003 \\
$\mathrm{~W}$ & 2.0163 & 0.1421 & 0.0019 & 0.0001 \\
$\mathrm{P}$ & 2.0021 & 0.1407 & 0.0004 & 0.0001 \\
\hline Laplace & shocks & & & \\
\hline $\mathrm{Y}$ & 1.6250 & 0.1074 & 0.0041 & 0.0001 \\
$\mathrm{C}$ & 1.4238 & 0.0907 & 0.0015 & 0.0001 \\
$\mathrm{I}$ & 1.8777 & 0.1295 & 0.0044 & 0.0002 \\
$\mathrm{E}$ & 1.2660 & 0.0783 & 0.0076 & 0.0003 \\
$\mathrm{~W}$ & 1.8375 & 0.1258 & 0.0018 & 0.0001 \\
$\mathrm{P}$ & 1.5168 & 0.0983 & 0.0004 & 0.0001 \\
\hline
\end{tabular}

Table 6: Simulated growth-rate distributions in the NK (second-order approximation): Estimated EP parameters. Legend: (Y) real GDP, (C) consumption, (I) investment, (E) employment, (P) inflation, (W) real wage. 


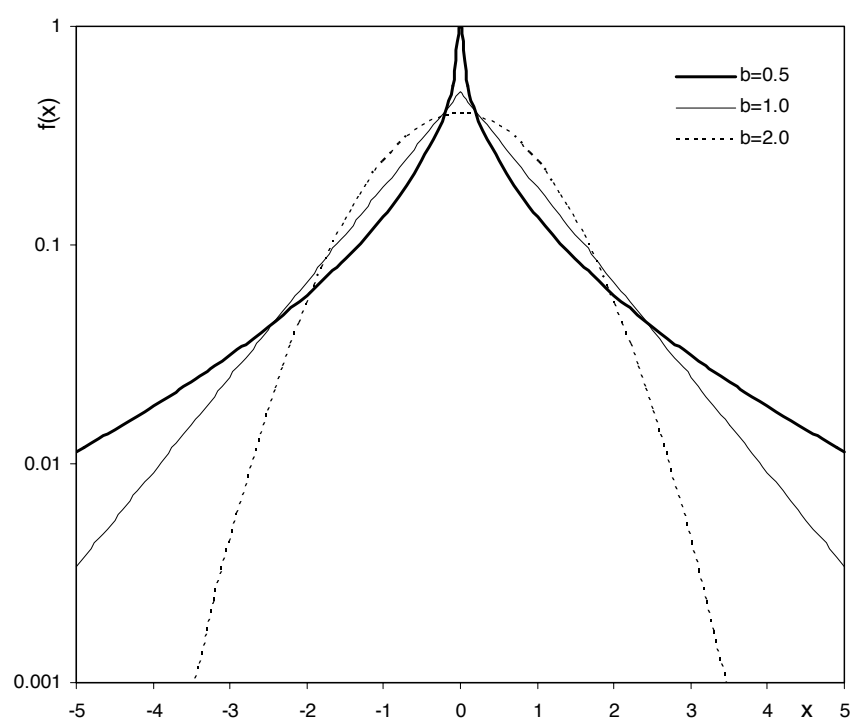

Figure 1: The exponential-power (EP) density for $m=0, a=1$ and different shape-parameter values: (i) $b=2$ : Gaussian density; (ii) $b=1$ : Laplace density; (iii) $b=0.5$ : EP with super-Laplace tails. Note: Log scale on the y-axis

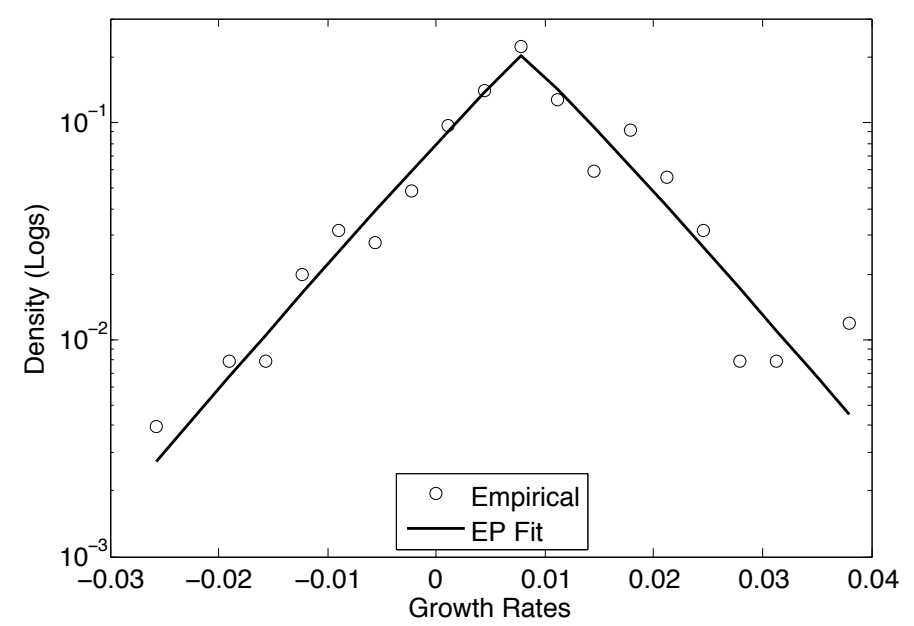

Figure 2: Binned empirical densities of U.S. real GDP growth-rate time-series distribution vs. ExponentialPower fit. 


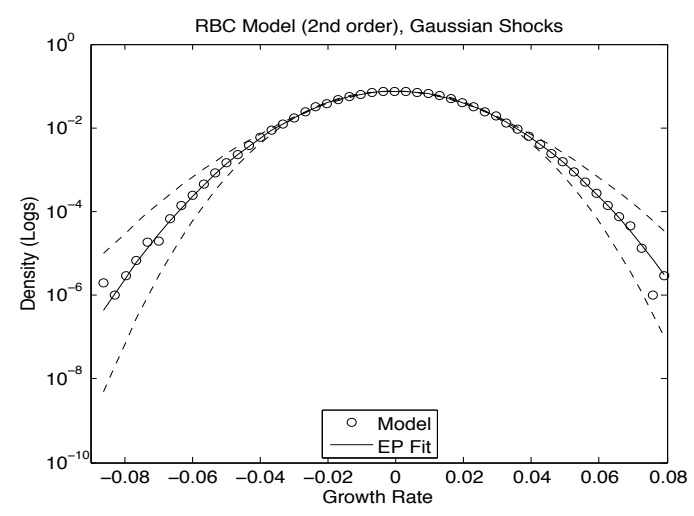

(a) RBC model, Gaussian shocks

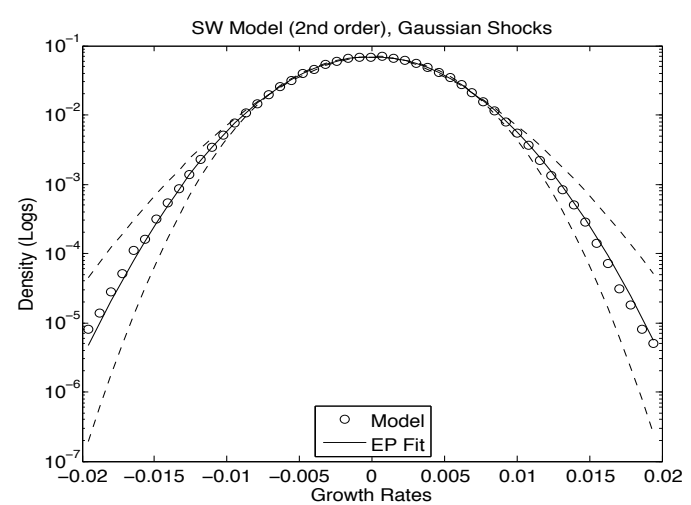

(c) SW model, Gaussian shocks

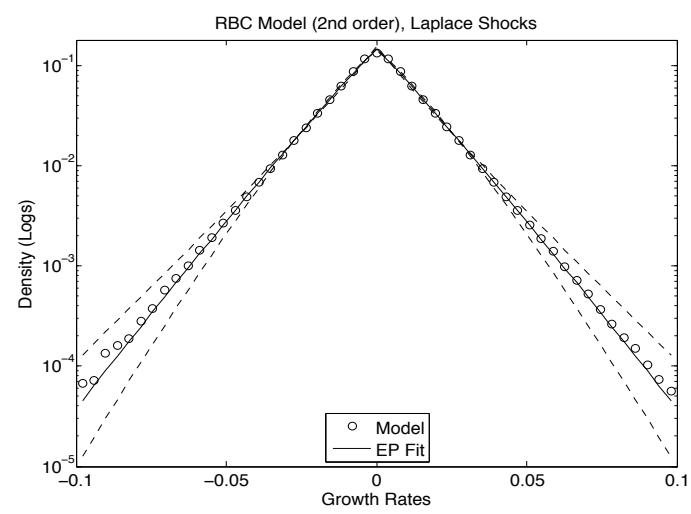

(b) RBC model, Laplace shocks

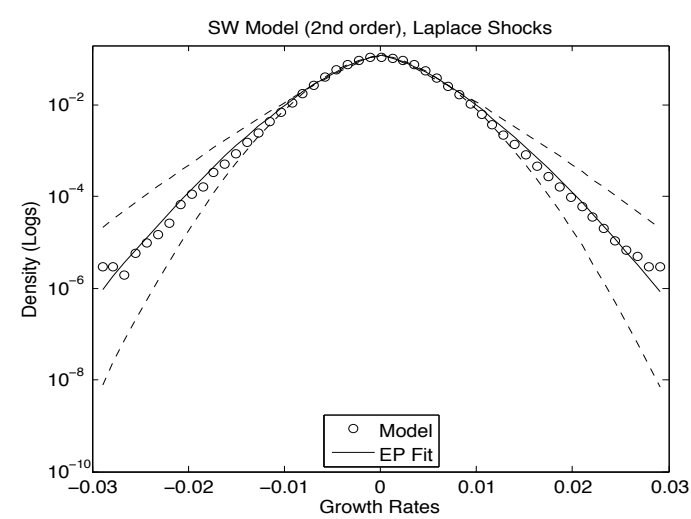

(d) SW model, Laplace shocks

Figure 3: Binned densities of simulated output growth-rate distributions in RBC and SW models under Gaussian or Laplace shocks. Solid line: Exponential-Power fit. Dashed lines: $\hat{b} \pm 2 \sigma(\hat{b})$ Cramér-Rao confidence intervals. 